\title{
Arginine-vasopressin infusion in a child with cranial diabetes insipidus during hyperhydration therapy with chemotherapy: a therapeutic challenge
}

\author{
Janani Devaraja 1 1, Sarah Sloan¹, Vicki Lee² and Paul Dimitri³ \\ 'Sheffield Children's NHS Foundation Trust, Sheffield, UK, 2Department of Paediatric Oncology, Sheffield Children's \\ NHS Foundation Trust, Sheffield, UK, and 3Department of Paediatric Endocrinology, Sheffield Children's NHS \\ Foundation Trust, Sheffield, UK
}

Correspondence

should be addressed

to J Devaraja

Email

janani.devaraja@nhs.net

\section{Summary}

An 11-year-old girl presented with acute lower limb weakness, dehydration, hypernatraemia and secondary rhabdomyolysis on a background of an 8-month history of polyuria. Radiological investigations revealed a suprasellar tumour which was diagnosed on biopsy as a non-metastatic germinoma. Further endocrinological investigations confirmed panhypopituitarism and she commenced desmopressin, hydrocortisone and thyroxine. Her chemotherapeutic regime consisted of etoposide, carboplatin and ifosfamide, the latter of which required 4 litres of hyperhydration therapy daily. During the first course of ifosfamide, titration of oral desmopressin was trialled but this resulted in erratic sodium control leading to disorientation. Based on limited literature, we then trialled an arginine-vasopressin (AVP) infusion. A sliding scale was developed to adjust the AVP dose, with an aim to achieve a urine output of 3-4 mL/kg/h. During the second course of ifosamide, AVP infusion was commenced at the outset and tighter control of urine output and sodium levels was achieved. In conclusion, we found that an AVP infusion during hyperhydration therapy was required to achieve eunatraemia in a patient with cranial diabetes insipidus. Developing an AVP sliding scale requires individual variation; further reports/case series are required to underpin practice.

\section{Learning points:}

- Certain chemotherapeutic regimens require large fluid volumes of hyperhydration therapy which can result in significant complications secondary to rapid serum sodium shifts in patients with diabetes insipidus.

- The use of a continuous AVP infusion and titrating with a sliding scale is more effective than oral desmopressin in regulating plasma sodium and fluid balance during hyperhydration therapy.

- No adverse effects were found in our patient using a continuous AVP infusion.

- Adjustment of the AVP infusion rate depends on urine output, fluid balance, plasma sodium levels and sensitivity/ response of the child to titrated AVP doses.

\section{Background}

Central diabetes insipidus (CDI) is a disease caused by arginine-vasopressin (AVP) deficiency leading to polyuria and polydipsia (1). The destruction of neurons in the paraventricular and supraoptic nuclei of the posterior pituitary gland leads to AVP deficiency (1). Causes of CDI include germinomas, craniopharyngiomas, trauma, and $20-50 \%$ of cases are idiopathic (1).

The initial presentation of a child with a germinoma is often a protracted history of evolving endocrine deficiencies (2). Most initially develop CDI followed by 
other endocrine deficiencies such as hypocortisolism, hypothyroidism and growth failure. CDI is treated with desmopressin, which is a synthetic analogue of AVP (1). Doses required to manage diuresis vary greatly between patients. The outcome with treatment is favourable with more than $85 \%$ overall 5 -year survival (2). However, the endocrine deficiencies do not resolve completely with tumour resolution and the children are often on lifelong hormone replacement therapy.

Certain chemotherapeutic agent protocols used in the treatment of suprasellar tumours require large fluid volumes (hyperhydration therapy) to reduce the risk of nephrotoxicity and haemorrhagic cystitis (3). Patients receiving hyperhydration therapy require strict monitoring of fluid balance and electrolytes to avoid complications secondary to sodium and potassium imbalance. Having a diagnosis of CDI while undergoing hyperhydration therapy presents an additional unique challenge. A study by Afzal et al. (4) showed that in children where cisplatin and/or ifosfamide chemotherapy were used (and hyperhydration therapy was therefore required), having CDI was a risk factor for prolonged admissions and complications including seizures, transient encephalopathy, and hyperreflexia with tremor. All children with CDI in their study also required daily changes in dosage and schedule of desmopressin.

In our patient, titration of oral desmopressin was insufficient to manage her fluid and electrolyte balance. We, therefore, used a continuous AVP infusion on a personalised sliding scale to successfully control her fluid balance and thus serum sodium concentration.

\section{Case presentation}

We report on an 11-year-old girl who had been previously well. Eight months prior to presentation, she developed polyuria, polydipsia and nocturia. Six months later, she developed frequent headaches. She then presented acutely to the paediatric team at a District General Hospital with generalised weakness and an inability to mobilise. Polyuria and polydipsia had resolved in the $24 \mathrm{~h}$ prior to presentation.

\section{Investigation}

She was found to have a serum sodium of $173 \mathrm{mmol} / \mathrm{L}$ (133-144 mmol/L), serum osmolality of $365 \mathrm{mosm} / \mathrm{kg}$ (275-295 mosm $/ \mathrm{kg})$, urine osmolality of $356 \mathrm{mosm} / \mathrm{kg}$ and creatinine kinase of 1825 units/L (30-170 units/L). A CT Head showed a large suprasellar mass.
She was transferred to our tertiary endocrine unit the following day under the care of the neurosurgery and endocrinology teams. MRI of brain showed a $3 \times 3$ $\mathrm{cm}$ mass involving the optic chiasm, optic nerves and hypothalamus with perilesional oedema (Fig. 1A and B). Biopsy of the mass revealed this to be a non-metastatic germinoma.

Endocrinological bloods demonstrated the following results: cortisol $<22 \mathrm{nmol} / \mathrm{L}$ at 08:00 h $(80-580 \mathrm{nmol} / \mathrm{L})$,
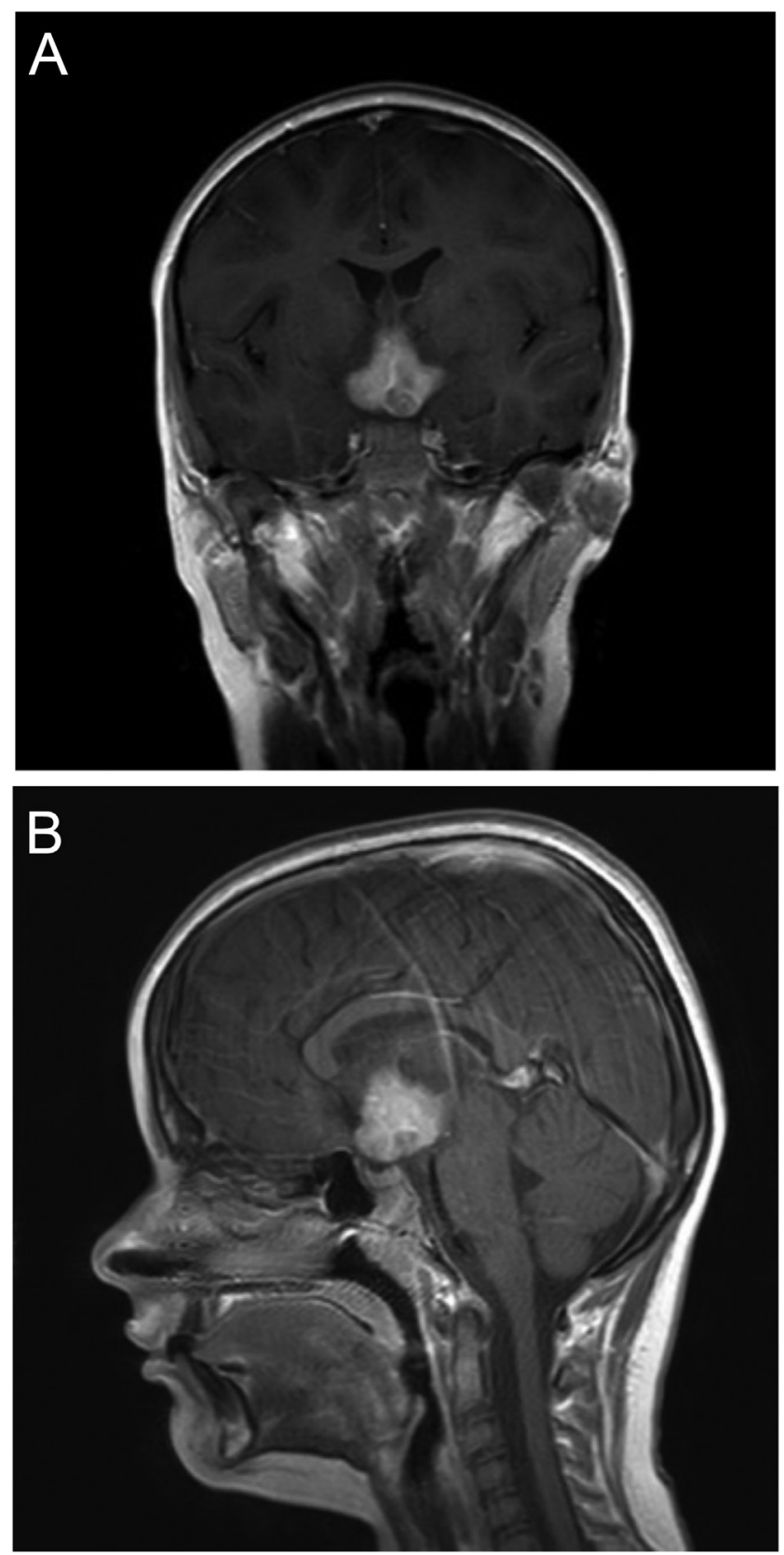

Figure 1

A coronal (A) and sagittal (B) MRI image of the germinoma. 
ACTH $<1.0 \mathrm{ng} / \mathrm{L}, \mathrm{TSH} 3.09 \mathrm{mU} / \mathrm{L}(0.5-3.6 \mathrm{mU} / \mathrm{L})$ and free T4 $6.6 \mathrm{pmol} / \mathrm{L}(10-16.9 \mathrm{pmol} / \mathrm{L})$ at $18: 00 \mathrm{~h}$ and IGF-1 $82 \mu \mathrm{g} / \mathrm{L}$ at $11: 00 \mathrm{~h}(160-581 \mu \mathrm{g} / \mathrm{L})$ thus leading to the diagnosis of panhypopituitarism.

An ophthalmology review revealed a severe restriction in visual acuity (right eye 6/85, left eye 6/38), reduced colour vision (right eye 15/17, left eye 16/17), and temporal optic disc pallor.

\section{Treatment}

Our patient was started on $0.9 \%$ saline at maintenance plus 5\% deficit to support rehydration, with urine output also replaced $\mathrm{mL}$ for $\mathrm{mL}$ with $0.9 \%$ saline. A brief course of dexamethasone was commenced to reduce intracranial oedema surrounding the tumour. At this point, the polyuria reoccurred, thereby showing that the 'initial resolution' of polyuria was due to cortisol deficiency. She was started on a titrating dose of desmopressin (0-75 $\mu \mathrm{g}$ BD), $50 \mu \mathrm{g}$ thyroxine and $10 \mathrm{mg} / \mathrm{m}^{2} /$ day hydrocortisone in three divided doses. With this treatment, her sodium levels gradually improved over the next 7 days. She was found clinically to have a degree of hypodipsia which persisted after the resolution of the intracranial oedema.
Hence her parents were advised to ensure that she took her maintenance fluid requirement daily.

The tumour was not amenable to surgery and she was started on alternating cycles of carboplatin/etoposide (cycles 1 and 3) and ifosfamide/etoposide (cycles 2 and 4). The two ifosfamide/etoposide cycles required $4 \mathrm{~L}$ of hyperhydration fluids daily using $0.45 \%$ saline $/ 2.5 \%$ dextrose with $20 \mathrm{mmol} / \mathrm{L}$ potassium chloride. In the first cycle, due to concerns about water intoxication we omitted desmopressin while allowing her to drink freely in addition to hyperhydration therapy. However, this led to a diuresis of $7 \mathrm{~L}$ on the first day resulting in lethargy due to polyuria preventing sleep, and she required additional intravenous fluid to replace the fluid deficit. The following day 25-50 $\mu \mathrm{g}$ doses of oral desmopressin given at the beginning of diuresis were trialled, which led to significant swings in her plasma sodium (Fig. 2) causing disorientation.

Based on a single publication reporting the management of 2 patients with CDI requiring hyperhydration therapy (5) we elected to trial an AVP infusion. A 0.04 units/mL AVP solution was prepared by adding 2 units of AVP (0.1 mL from 20 unit/mL ampoule) to $49.9 \mathrm{~mL}$ of $0.9 \%$ saline.

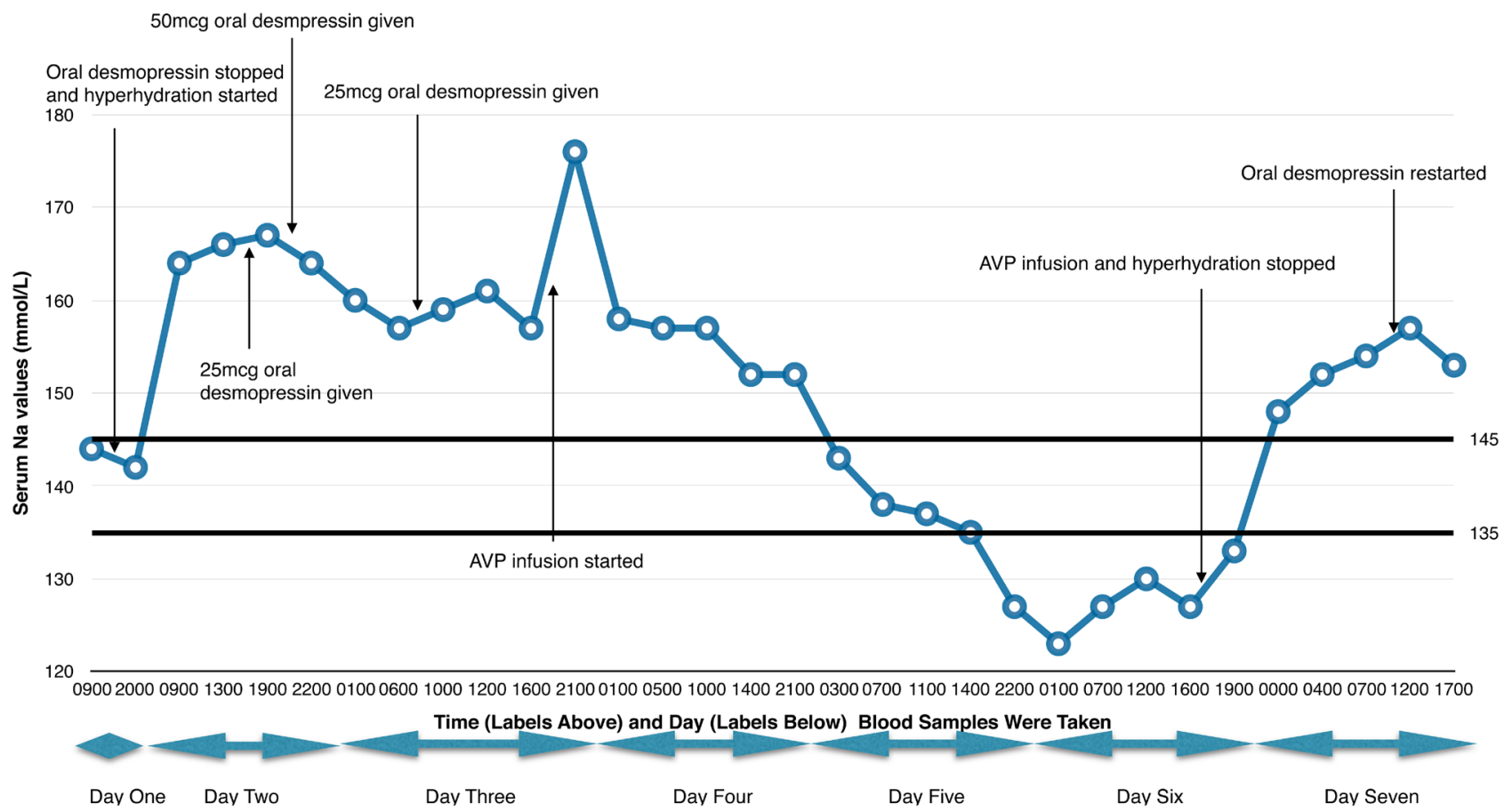

Figure 2

Serum sodium ( $\mathrm{mmol} / \mathrm{L}$ ) levels during first chemotherapy cycle involving hyperhydration. The normal serum sodium ranges are 135-145 mmol/L. The timing of blood samples was rounded to the nearest hour. 
Table 1 The AVP (arginine vasopressin) infusion sliding scale initially used.

\begin{tabular}{l}
$\begin{array}{l}\text { Urine output, } \\
\mathbf{m L / k g / h}\end{array}$ \\
\hline$<2$ \\
$2-3$ \\
$3-4$ \\
$4-5$ \\
$>5$ \\
\hline
\end{tabular}

\section{Action taken}

Stop infusion till urine output is 3-4 mL/kg/h Reduce infusion rate by $50 \%$ Maintain infusion rate Increase infusion rate by $50 \%$ Increase infusion rate by $100 \%$

Urine output of 3-4 mL/kg/h was targeted.

A starting dose of 0.0001 units $/ \mathrm{kg} / \mathrm{h}$ was used and we created an 'AVP sliding scale' in order to titrate the AVP infusion based on urine output (initially aiming for 3-4 $\mathrm{mL} / \mathrm{kg} / \mathrm{h}$ to compensate for the large amount of infused fluid). The outline of the initial sliding scale we used is attached (Table 1). She was catheterised and her urine output monitored hourly.

The original protocol required further development in response to her clinical condition. Initially, we adjusted the AVP infusion rate by $50 \%$ depending on the urine output. However, we found that sizeable dose changes caused large swings in her serum sodium and therefore the rate change was reduced to $25 \%$. Furthermore, the target urine output required adjustment based on fluid balance. When the AVP infusion was initially commenced during the first ifosfamide/etoposide cycle, she was hypernatraemic with a large negative fluid balance. Therefore the target urine output was reduced to $2-3 \mathrm{~mL} / \mathrm{kg} / \mathrm{h}$ until sodium levels normalised. With the second ifosamide/etoposide cycle, target urine output of $3-4 \mathrm{~mL} / \mathrm{kg} / \mathrm{h}$ led to rapid decline in sodium levels due to large intravenous fluid intake. Therefore target urine output was increased to $5-6 \mathrm{~mL} / \mathrm{kg} / \mathrm{h}$ to counteract this and maintain a neutral fluid balance.

\section{Outcome and follow-up}

During the first ifosfamide cycle, our patient's plasma sodium showed significant fluctuation (Fig. 2). During the second cycle, AVP infusion was started at the onset and thus tighter control of plasma sodium was achieved (Fig. 3) and she did not experience any related symptoms.

Following three cycles of chemotherapy, intracranial MRI demonstrated a tiny suprasellar residuum. Visual acuity was $6 / 45$ in the right eye and $6 / 15$ in the left, an improvement from $6 / 85$ and $6 / 38$ prior to starting chemotherapy.

She received proton therapy at University Hospital Essen in Germany, and following this there was further regression of the focal abnormality on her cranial MRI, and further improvement in her visual acuity (6/30 in right eye, $6 / 15$ in left eye).

Both growth hormone $(\mathrm{GH})$ stimulation with clonidine $(200 \mu \mathrm{g})$ and LHRH testing showed suboptimal responses. She was started on GH and will soon commence pubertal induction with transdermal oestrogen. She continues to be monitored by the endocrinology and oncology teams, and remains well on desmopressin $75 \mu \mathrm{g} / 75 \mu \mathrm{g} / 100 \mu \mathrm{g}$ daily, levothyroxine $75 \mu \mathrm{g}$ daily, hydrocortisone $10 \mathrm{mg} /$ $\mathrm{m}^{2}$ daily in three divided doses and GH $0.7 \mathrm{mg} / \mathrm{m}^{2}$ daily.

\section{Discussion}

Our patient had symptoms suggestive of diabetes insipidus for several months prior to admission. She had also developed hypodipsia which potentially precipitated her decompensated diabetes insipidus. The polyuria had appeared to 'resolve' just before she presented but its resumption once hydrocortisone was commenced demonstrated that progression to cortisol deficiency had masked her diabetes insipidus. The 'masking' of diabetes insipidus is due to impact of cortisol deficiency on AVP dependent and AVP independent mechanisms in the distal convoluting tubules and collecting ducts of the kidney, leading to reduced water clearance (6).

Hyperhydration presents a challenge when patients requiring chemotherapy also have CDI. This challenge was further compounded in our patient due to impaired thirst, which leads to a higher risk of decompensation. We found that by using an AVP infusion during hyperhydration therapy, we achieved better control of our patient's serum sodium and fluid balance. She also felt less lethargic and was better able to cope with the symptoms relating to chemotherapy and she suffered no adverse effects.

There is a limited literature of case series on the use of AVP infusions in paediatric and adult patients. WiseFaberowski et al. (7) used a continuous AVP infusion in 18 children with pre- and post-operative DI and compared their findings with 19 historical controls. They were able to maintain the children's plasma sodium levels within range much more effectively with a continuous AVP infusion. No adverse effects were detected. However, none of these patients required hyperhydration therapy. Levine et al. (8) used an AVP infusion in adult patients with cranial lymphoma who did receive hyperhydration therapy with methotrexate. They initially trialled oral and subcutaneous desmopressin in one patient, and similar to us they found variable fluctuation in plasma sodium levels. Their protocol used a more dilute infusion $(0.005$ units $/ \mathrm{mL}$ vs 


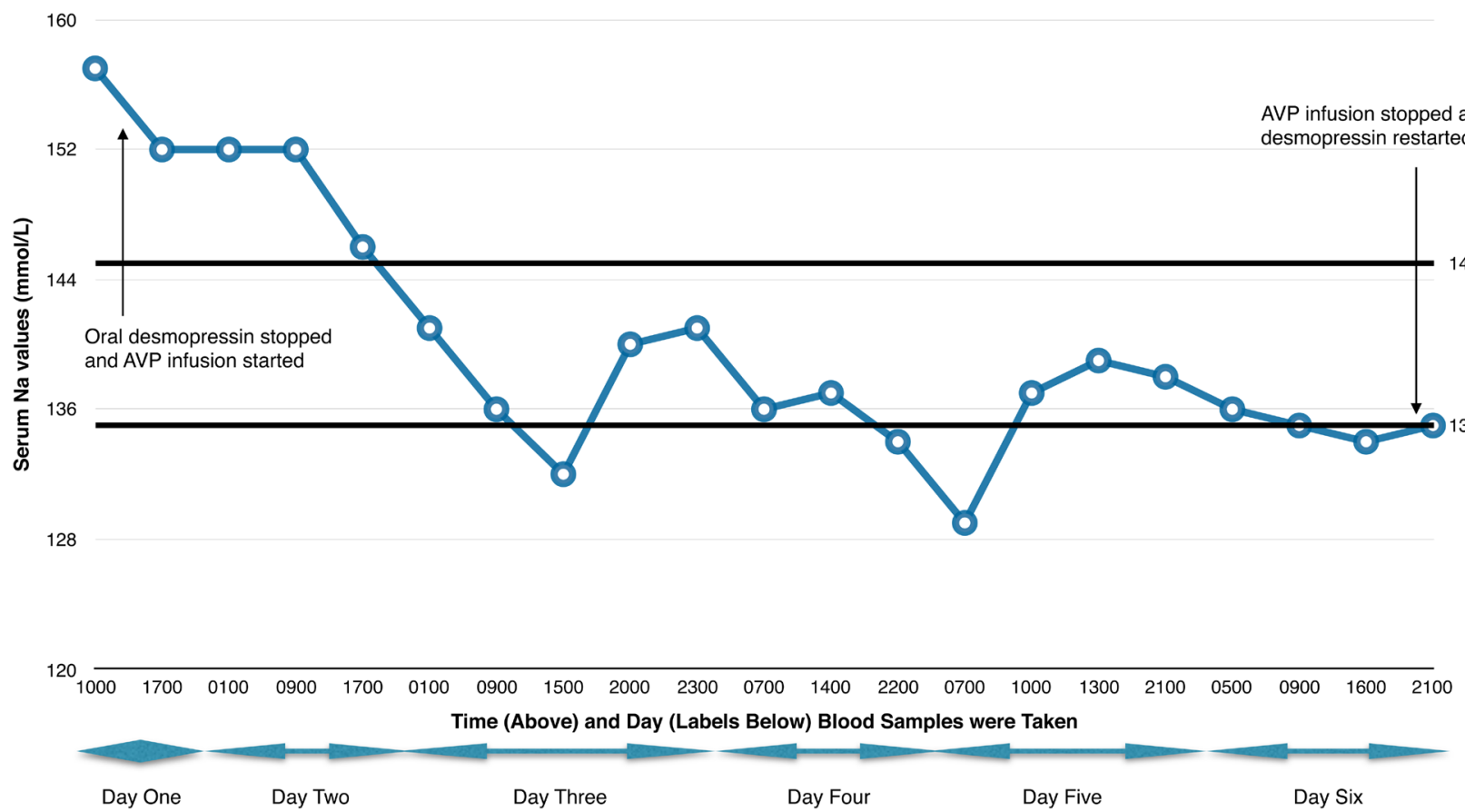

Figure 3

Serum sodium ( $\mathrm{mmol} / \mathrm{L}$ ) levels during second chemotherapy cycle involving hyperhydration. The normal serum sodium ranges are $135-145 \mathrm{mmol} / \mathrm{L}$. The timing of blood samples was rounded to the nearest hour.

0.04 units $/ \mathrm{mL}$ ) and a different titration method (titration by $50-100 \%$ ) compared to ours. They also found it more effective to maintain plasma sodium within range with a continuous AVP infusion and neither of their two patients had side effects. Most pertinent to our case, Bryant et al. (5) trialled an AVP infusion in two children with DI and suprasellar germinoma, and compared with one child in whom desmopressin was withheld, all of whom required the same chemotherapy and hyperhydration regime. Our protocol for preparing an AVP infusion and the starting dose was adapted from theirs. They found that by using an AVP infusion, better control of the fluid and electrolyte balance was achieved. The children also required less fluid (the child who did not receive an AVP infusion required $20 \mathrm{~L} / \mathrm{m}^{2} /$ day of fluids compared to the other two children who received $3.8 \mathrm{~L} / \mathrm{m}^{2} /$ day).

Currently, there are no clinical trials in literature looking at continuous AVP infusions in patients with CDI requiring hyperhydration, probably due to rarity of this occurrence. Therefore it seems likely that management of these patients will depend on individual case reports or small case series. Based on our experience and the current literature, in the absence of other effective alternatives we recommend the use of continuous AVP infusions in the management of children with CDI requiring chemotherapy that necessitates hyperhydration. Using a continuous AVP infusion enables a tighter control of plasma sodium levels to be achieved through regulating urine output, and thus avoids the clinical effects that are seen with sharp swings in serum sodium. AVP infusions also have a rapid onset and termination profile and thus rate titrations lead to a rapid clinical effect. AVP infusions have a good safety profile with no adverse effects. Levine et al. (7) recognised hypertension as a possible adverse effect, however, the doses used to manage DI are significantly smaller $(<0.5 \%)$ than the doses used to manage hypotension in adults.

Based on our experience, we recommend the following steps to be taken when starting a child on continuous AVP infusion for hyperhydration therapy:

1. Prepare AVP infusion by adding 2 units $(0.1 \mathrm{~mL}$ from 20 unit/mL ampoule) to $49.9 \mathrm{~mL} 0.9 \%$ saline or $5 \%$ dextrose to make up $50 \mathrm{~mL}$ syringe. Solution is therefore 0.04 units $/ \mathrm{mL}$. If a more concentrated infusion is required, then five units of AVP $(0.25 \mathrm{~mL})$ added to $49.75 \mathrm{~mL}$ solution to give 0.1 units $/ \mathrm{mL}$ could be used (5).

2. Start AVP infusion at the onset of hyperhydration therapy. Omit the usual oral desmopressin doses for the duration of AVP infusion.

3. HDU (High Dependency Unit) admission is recommended. 
Table 2 Recommended protocol using the AVP (arginine vasopressin) Infusion Sliding Scale.

\begin{tabular}{l}
$\begin{array}{l}\text { Urine output, } \\
\mathbf{m L / k g} / \mathbf{h}\end{array}$ \\
\hline$<2$ \\
$2-3$ \\
$3-4$ \\
$4-5$ \\
$>5$
\end{tabular}

\section{Action taken}

Stop infusion till urine output is $3-4 \mathrm{~mL} / \mathrm{kg} / \mathrm{h}$

Reduce infusion rate by $25 \%$

Maintain infusion rate

Increase infusion rate by $25 \%$

Increase infusion rate by $50 \%$

Aim for urine output 3-4 mL/kg/h, however, this target will be dependent on fluid balance. Calculate hourly urine output to enable titration of AVP to be done hourly. Size of increment/decrement may need to be altered depending on patient's sensitivity to desmopressin.

4. Some children may need a separate cannula inserted if the AVP infusion is incompatible with their chemotherapy drugs.

5. Insert a urinary catheter in all patients to enable hourly monitoring of urine output and fluid balance. Urea and electrolytes should be measured 6 hourly and the child should be weighed daily if possible.

6. Aim for a urine output of $3-4 \mathrm{~mL} / \mathrm{kg} / \mathrm{h}$. However, if there is a significantly negative fluid balance as a result, aim for a lower urine output during AVP titrations. Conversely, if there is significantly positive fluid balance, target a higher urine output.

7. Start the AVP infusion at 0.0001 units $/ \mathrm{kg} / \mathrm{h}$ and alter the rate according to our recommended protocol (Table 2). The size of increment/decrement may need to be bigger or smaller depending on the sensitivity of the patient to desmopressin. We recommend that titration is performed hourly.

8. Once therapy is complete and serum sodium is within the normal range for the patient, oral desmopression should be recommenced $1 \mathrm{~h}$ prior to discontinuing the AVP infusion.

We recognise that admission to a specialist HDU may be challenging in some clinical settings; however, with the intensity of observations and hourly titrations needed, 1:1 nursing care is recommended. We also acknowledge the limitation of extrapolating an AVP infusion sliding scale based on a single patient and limited experience in published literature. Thus we recommend that further experience in using this protocol or alternatives published in the literature would aid in refining the sliding scale and management of patients with CDI undergoing chemotherapeutic regimens requiring hyperhydration.

\section{Declaration of interest}

The authors declare that there is no conflict of interest that could be perceived as prejudicing the impartiality of the research reported.

\section{Funding}

This research did not receive any specific grant from any funding agency in the public, commercial or not-for-profit sector.

\section{Patient consent}

A written consent has been obtained from the patient's parents and are attached to the submission.

\section{Author contribution statement}

Dr V Lee is the patient's Paediatric Oncology Consultant and Prof P Dimitri is her Paediatric Endocrinology Consultant and managed this patient throughout the course of her therapy. J Devaraja is a Paediatric Registrar who had been involved with the endocrinological care of the patient when she was initially admitted, and during her chemotherapy regimens. J Devaraja and S Sloan wrote the manuscript. P Dimitri and V Lee reviewed and contributed to the content of the manuscript. P Dimitri is the senior author.

\section{References}

1 Di Iorgi N, Napoli F, Allegri AE, Olivieri I, Bertelli E, Gallizia A, Rossi A \& Maghnie M. Diabetes insipidus - diagnosis and management. Hormone Research in Paediatrics 201277 69-84. (https://doi.org/10.1159/000336333)

2 Osorio DS \& Allen JC. Management of CNS germinoma. CNS Oncology 20154 273-279. (https://doi.org/10.2217/cns.15.13)

3 Baxter Healthcare Ltd. Ifosfamide Injection $1 \mathrm{~g}$ - summary of Product Characteristics. (available at: https://www.medicines.org.uk/emc/pr oduct/1834/smpc). Accessed on 9th July 2020.

4 Afzal S, Wherrett D, Bartels U, Tabori U, Huang A, Stephens D \& Bouffet E. Challenges in management of patients with intracranial germ cell tumor and diabetes insipidus treated with cisplatin and/ or ifosfamide based chemotherapy. Journal of Neurooncology 201097 393-399. (https://doi.org/10.1007/s11060-009-0033-z)

5 Bryant WP, O’Marcaigh AS, Ledger GA \& Zimmerman D. Aqueous vasopressin infusion during chemotherapy in patients with diabetes insipidus'. Cancer 199474 2589-2592. (https://doi.org/10.1002/10970142(19941101)74:9<2589::aid-cncr2820740929>3.0.co;2-6)

6 Non L, Brito D \& Anastasopoulou C. Neurosarcoidosis-associated central diabetes insipidus masked by adrenal insufficiency. BMJ Case Reports 20152015 bcr2014206390. (https://doi.org/10.1136/bcr-2014-206390)

7 Wise-Faberowski L, Soriano SG, Ferrari L, McManus ML, Wolfsdorf JI, Majzoub J, Scott RM, Truog R \& Rockoff MA. Perioperative management of diabetes insipidus in children. Journal of Neurosurgical Anesthesiology 200416 220-225. (https://doi.org/10.1097/00008506200407000-00006)

8 Levine JA, Karam SL, O'Connor C, Kumar S, Soundarrajan M, McConnell D, Ammar AT, Gale AM, Zimmerman D \& Szmuilowicz ED. Central diabetes insipidus and chemotherapy: use of a continuous arginine vasopressin infusion for fluid and sodium balance. AACE Clinical Case Reports 20184 e487-e492. (https://doi. org/10.4158/ACCR-2018-0165)

Received in final form 18 August 2020

Accepted 25 January 2021 